\section{Workflow Management Systems}

Mor Peleg

Department of Information Systems, University

of Haifa, Haifa, Israel

\section{Synonyms}

Business-process management systems; Workflow management system

\section{Definition}

According to the Workflow Management Coalition, a workflow management system is a system that completely defines, manages, and executes workflows through the execution of software whose order of execution is driven by a computer representation of the workflow logic.

\section{Workflow Model}

A workflow model includes two parts: a model of the workflow process, including the activities, their order, and their start and termination conditions, and a model of the organization at which the workflow takes place, including the individual actors, the organizational roles, and departmental units who all perform workflow activities. A workflow process is performed for cases that trigger it (e.g., item order, a candidate's application). A workflow task performed for a specific case is called "workflow item." Workflow items are performed by a participant: an organizational actor, role, unit, or resource. An activity is a work item performed by a participant. As stated by Wil van der Aalst, "Workflow management is the glue between the cases, the tasks, and the organization."

\section{Cross-References}

> Executable Knowledge

\section{Recommended Reading}

1. van der Aalst WMP. The application of Petri nets to workflow management. J Circuits Syst Comput. 1998;8(1):21-66. 